\title{
Child and parent training sessions led to improved child behaviour in child conduct disorder
}

Webster-Stratton C,Hammond M Treating children with early-onset conduct problems: a comparison of child and parent training interventions. J Consult Clin Psychol 1997 Feb,65:93-109.

\section{Objective}

To compare child training, parent training, and a combined training programme for the treatment of children with conduct problems.

\section{Design}

Randomised controlled trial with 1 year follow up.

\section{Setting}

Parenting clinic at the University of Washington, Seattle, Washington, USA.

\section{Patients}

97 children who were 4 to 7 years of age (mean age 5.7 y, $74 \%$ boys) and who were referred for conduct problems lasting for at least 6 months. Children had a Diagnostic and Statistical Manual of Mental Disorders, 3rd edition, revised diagnosis of oppositional defiant disorder and conduct disorder. 166 parents (95 mothers and 71 fathers) participated.

\section{Intervention}

Participants were allocated to child training (CT) $(n=27$ children), parent training (PT) $(\mathrm{n}=26$ children and 43 parents), combined child and parent training $(\mathrm{CT}+\mathrm{PT})(\mathrm{n}=22$ children and 36 parents), or a wait list control $(\mathrm{n}=22$ children and 40 parents). The programmes were weekly 2 hour sessions that lasted about 6 months. The CT programme included videotaped vignettes and fantasy play that addressed interpersonal difficulties. The PT programme included videotaped programmes on parenting and interpersonal skills. Wait list control group families waited 9 months and then were allocated to 1 of the 3 programmes.

\section{Main outcome measures}

Outcomes relating to clinical significance: $t$ score $<60$ on the mothers' Child Behaviour Checklist; and reductions of $\geqslant 30 \%$ in child negative behaviour as reported by mothers (Parent Daily Report), in mother criticisms (Dyadic Parent-Child Interactive Coding System - revised [DPICS-R]) and in total child deviance (DPICS-R).

\section{Main results}

Mothers' reports from the treatment groups that included PT showed improvements in their child's behaviour. $81 \%$ of mothers in the PT group and 70\% of mothers in the CT + PT group reported improvement compared with $27 \%$ of mothers in the control group $(\mathrm{p}<0.01)$. The CT and control groups did not differ $(37 \% v 27 \%)$. A reduction of $\geqslant 30 \%$ in targeted child negative behaviour occurred in $89 \%$ of children in the CT group, $92 \%$ of children in the PT group, and $91 \%$ of children in the $\mathrm{CT}+\mathrm{PT}$ group. All 3 treatments differed from the control $(\mathrm{p}<0.001)$. A reduction of $\geqslant 30 \%$ in deviant behaviour occurred in 73\% of children in the CT group, $73 \%$ of children in the PT group, $60 \%$ of children in the CT + PT group, and 55\% of children in the control group. None of the treatment groups differed from the control group. A reduction of $\geqslant 30 \%$ in criticism from mothers occurred to the greatest extent in the CT + PT group $(71 \%)$ and the PT group (68\%). The target reduction was reported for $46 \%$ of mothers in the CT group and $28 \%$ of mothers in the control group $(\mathrm{p}<0.05$ for PT and $\mathrm{CT}+\mathrm{PT} v$ control).

\section{Conclusions}

Improvement in child behaviour was shown after weekly sessions of child or parent training, or a combined programme. The combined approach showed improvement in some measures for which the single interventions did not.

Source of funding: National Institute of Mental Health.

For article reprint: Professor C Webster-Stratton, Parenting Clinic, Box 354801, School of Nursing, University of Washington, Seattle, WA 98195, USA. Fax +1 2065436040.

\section{Commentary}

This trial by Webster-Stratton and Hammond contributes to growing evidence that shows the efficacy of parent training for families of children with disruptive behaviour disorders. These appear to be robust interventions: different variations of parent training programmes based on behaviour have proved effective for children with oppositional disorders, conduct disorders, ${ }^{1}$ and attention deficit hyperactivity disorders. ${ }^{2}$

This study shows that the outcome of parent training can be improved by working directly with children. While child training did not increase the effect of parent training on child management skills and behaviour problems, child training showed improvement in areas that are not influenced by parent training (eg, interactions with peers). The amount of improve- ment evident, proportion of families benefiting, dimensions of parent and child functioning affected, and stability of these improvements at follow up suggest that these are clinically meaningful outcomes.

This is an affordable and logistically feasible programme. Treatment manuals and videotapes allow this programme to be disseminated to other settings. As previous studies have shown, parent training was done successfully in cost effective groups. ${ }^{3}$ Similarly, as a group programme, child training is an affordable addition to parent training. In addition to improving outcome, concurrent activities for children allow families who are unable to secure reliable child care to participate in parent training programmes.

With notable exceptions, parent training programmes do not consistently address problems at school. ${ }^{4}$ Future studies need to determine whether this programme reduces behaviour problems at school. If not, an effective home-school training component should be developed.

As the authors note, this is a demanding parent training protocol. While some families may not be ready for the parent training component, the child training programme represents a demonstrably useful alternative.

Charles Cunningham, $\mathrm{PhD}$ McMaster University Hamilton, Ontario, Canada

1 Kazdin AE. J Am Acad Child Adolesc Psychiatry 1997:36:1349-56.

2 Anastopoulos AD, Shelton TL, DuPaul GJ, et al. JAbnorm Child Psychol 1993;21:581-96.

3 Cunningham CE, Bremner R, Boyle M. J Child Psychol Psychiatry 1995;36:1141-59.

4 McNeil CB, Eyberg S, Eisenstadt TH, et al.J Clin Child Psychol 1991;20:140-51. 